\title{
Study of the Soft X-Ray Emission from Carbon Ions in a Capillary Discharge
}

\author{
Jorge J. Rocca, Member, IEEE, Mario C. Marconi, and F. G. Tomasel
}

\begin{abstract}
The soft X-ray emission from plasmas produced in polyacetal and polyethylene capillary discharges excited by current pulses 100 ns FWHM, 50 ns risetime, and peak currents up to $60 \mathrm{kA}$ has been studied. Time-resolved spectra show that intense CvI line emission dominates in the polyethylene discharges, peaking shortly after the maximum of the current and vanishing before the end of the current pulse. Polyacetal capillary spectra are dominated by Ovi lines and show weak Cvi emission. Plasma density and temperature for the polyethylene discharge were derived from the Stark broadening of CvI Balmer lines and the relative intensities of $\mathrm{CvI}$ and $\mathrm{Cv}$ lines. $\mathrm{A}$ short ( 5 ns FWHM) pulse of energetic beam electrons was detected in coincidence with the onset of the discharge. The results are discussed in relation to the possibility of obtaining soft $X$-ray amplification by plasma recombination in a capillary discharge plasma column.
\end{abstract}

\section{INTRODUCTION}

ARGE amplification of soft X-ray radiation has been demonstrated in plasmas created by high energy lasers [1]-[4]. Output powers up to several megawatts [5] and pulse energies up to $3 \mathrm{~mJ}[6]$ have been reported and the applications of these laser sources to X-ray holography and microscopy has been explored [7], [8]. However, more efficient and compact devices need to be developed. The utilization of smaller pump lasers in excitation schemes that could result in table-top soft $\mathrm{X}$-ray lasers is a topic of current research [9], [10].

We have proposed the possibility of developing compact and efficient soft $\mathrm{X}$-ray recombination lasers by direct excitation of a capillary plasma column with a fast discharge current pulse [11]. Capillary discharges are known to generate dense highly ionized plasmas with a large length-to-diameter ratio [12]-[15] in which the stabilizing effect of the capillary walls allows for good axial uniformity. Cooling of the plasma by electron heat conduction to the capillary walls and plasma radiation causes

\footnotetext{
Manuscript received September 27, 1991; revised March 24, 1992. This work was supported by the Department of Energy, Advanced Energy Projects Grant DE-F602-91ER12110 and by the National Science Foundation by Grant ECS 9013372 . The work of M. C. Marconi was supported by the Universidad Nacional de Buenos Aires and Consejo Nacional de Investigaciones Cientificas y Tecnicas de la Republica Argentina.

J. J. Rocca and M. C. Marconi are with the Department of Electrical Engineering, Colorado State University, Fort Collins, CO 80523.

F. G. Tomasel is with the Department of Electrical Engineering, Colorado State University, Fort Collins, CO 80523 , on leave from the Departmento de Fisica, Facultad de Ingeniera, Universidad Nacional de Mar del Plata, Argentina.

IEEE Log Number 9204363
}

the collisional recombination of highly ionized species into ions of lower charge, and could possibly produce population inversions and amplification. The symmetrically concave radial profile of the density in the capillary plasma could help to waveguide the $\mathrm{X}$-ray emission.

Of special interest is the generation of discharge-excited plasma columns which recombine into hydrogenand lithium-like ions, as these species have already been demonstrated to produce amplification in laser-created plasmas [2], [3]. In the first case, the collisional recombination of totally ionized atoms into highly excited levels of hydrogenic ions followed by collisional electron deexcitation generates a population inversion between the levels of the 3-2 transition.

In previous experiments, we have studied the extreme ultraviolet emission from a $500 \mu \mathrm{m}$ diameter $\mathrm{LiH}$ capillary excited by a $5 \mathrm{~J}, \mathbf{5 0} \mathrm{ns}$ FWHM current pulse of a $\mathbf{4 0}$ $\mathrm{kV}$ discharge. Increased emission at $72.9 \mathrm{~nm}$, the wavelength of the LiII 3-2 transition, was observed at the end of the current pulse, as can be expected from the excitation of this line following collisional recombination [15], [16]. However, in the case of the LiII $72.9 \mathrm{~nm}$ line, even if rapid plasma cooling to a temperature of $4 \mathrm{eV}$ could be achieved, the amplification would be limited to less than $0.1 \mathrm{~cm}^{-1}$ by the relatively low plasma density $N_{e} \leq 1$. $10^{17} \mathrm{~cm}^{-3}$ required to avoid the depletion of the population inversion by collisional electron deexcitation. The favorable scaling of the plasma density limit and the gain coefficient with the nuclear charge as $Z^{7}$ and $Z^{7.5}$ [17] favors the search for gain in ions of higher charge. Therein lies the interest in the generation of highly ionized carbon plasma columns.

In this paper we report the results of time-resolved and time-integrated spectroscopy of the soft $\mathrm{X}$-ray emission from polyethylene $\left(\mathrm{CH}_{2}\right)_{n}$ and polyacetal $\left(\mathrm{CH}_{2} \mathrm{O}\right)_{n}$ capillaries excited by current pulses of approximately $100 \mathrm{~ns}$ FWHM, 50 ns risetime and peak current up to $55 \mathrm{kA}$ from compact table-top discharges. Polyethylene was selected as the material to be studied because it offers the possibility of generating nearly pure carbon plasmas, the hydrogen being easily ionized in the core of the discharge. Polyethylene capillary discharges have been previously studied as sources of continuum radiation [12], and as incoherent soft X-ray sources for contact microscopy and lithography [13]. However, the temporal evolution of the soft X-ray radiation and its dependence on the discharge parameters has not been previously reported. 
Polyacetal discharges, which contain oxygen, can be expected to generate cooler plasmas [13] with a reduced CVII concentration. These discharges were nevertheless also studied in relation with the recent report by Steden and Kunze [18] of the observation of short bursts of radiation at $18.2 \mathrm{~nm}$ which increase in intensity with the capillary length in a low voltage $(7-16 \mathrm{kV})$ polyacetal capillary discharge. These bursts of radiation, which were detected to occur in the vicinity of the peak of the second half cycle of the current pulse with a scintillator-photomultiplier detector combination, were interpreted as amplified spontaneous emission. Our study of these discharges covered an extended voltage range, and the diagnostics included both scintillator-photomultiplier detection of the temporal evolution of the emission at specific wavelengths of interest and gated intensified array detector acquisition of the time-resolved soft X-ray spectra. The latter allows for the simultaneous monitoring of the intensities of several transitions and provides a clearer distinction between lines and background continuum radiation. Our study of polyacetal discharges could not identify the emission of bursts of radiation at $18.2 \mathrm{~nm}$ that could be associated with amplified stimulated emission. As discussed in Section $V$, the polyacetal spectra are found to be dominated by OVI lines and to produce only weak Cvı emission.

\section{The Capillary Discharges}

Two low inductance capillary setups have been constructed to conduct the experiments described herein. The first one, illustrated in Fig. 1, consists of a circular multilayer foil capacitor that is directly connected to the two graphite electrodes placed at the ends of an evacuated capillary channel. Each layer of the capacitor is composed of two aluminum foil disks, $0.025 \mathrm{~mm}$ thick, separated by three layers of mylar $0.075 \mathrm{~mm}$ thick each. Eight of these disk capacitors are stacked together and connected in parallel by alternately contacting the aluminum foil disks at the inner (high voltage) or outer (ground) edges to obtain a total capacitance of $100 \mathrm{nF}$. The entire stack of aluminum and mylar foils is pressed between an aluminum plate and an acrylic plate to achieve a close contact between the layers. The effective thickness of the capacitor is $3 \mathrm{~mm}$, and the total discharge circuit inductance is $18 \mathrm{nH}$ when a $1 \mathrm{~cm}$ long, $1 \mathrm{~mm}$ diameter capillary is used. The foil discharge capacitor was routinely operated at voltages up to $17 \mathrm{kV}$. Perforation of the dielectric occurring in the vicinity of the outer edge of the aluminum disks limited the operating voltages of the discharge to less than $20 \mathrm{kV}$. Assembly of the capacitor into transformer oil allowed us to increase the operation voltage up to $25 \mathrm{kV}$, generating a peak discharge current over $40 \mathrm{kA}$ through a $1 \mathrm{~mm}$ diameter, $1 \mathrm{~cm}$ long capillary.

The current pulse was monitored with a Rogowski coil having a risetime of less than 2 ns and the waveforms were recorded with a 250 megasamples per second transient digitizer. The capillaries were evacuated to a pres-
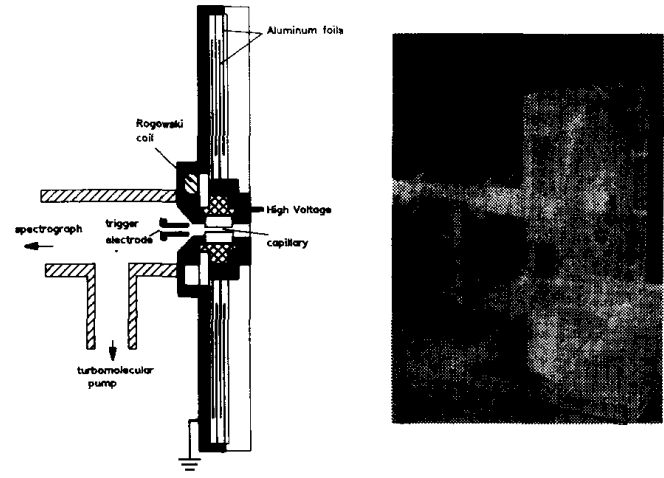

Fig. 1. Schematic diagram and photograph of the capillary discharge with a foil capacitor. For simplicity only two layers of the capacitor are shown.

sure below $1 \cdot 10^{-5}$ torr utilizing a turbomolecular pump and the capacitor was charged with a dc high voltage power supply to a voltage below the maximum hold off voltage of the capillary. In this setup the capillary itself acts as a discharge switch [12], [13], [15]. Surface flashover of the capillary channel is achieved by producing a secondary spark between the cathode, which is at ground potential, and a negative trigger electrode placed in close proximity.

To reach higher discharge currents a second set up was constructed. The structure of this discharge, which is illustrated in Fig. 2, utilizes ceramic capacitors but otherwise it is similar to the one described above. In this case the discharge capacitance of $100 \mathrm{nF}$ was obtained by connecting in parallel thirty two ceramic capacitors of $3.3 \mathrm{nF}$ nominal capacitance each, placed in the periphery of a rectangle $53 \mathrm{~cm}$ wide by $66 \mathrm{~cm}$ high having as center the capillary axis. The capacitors were directly connected to the capillary electrodes through a parallel plate transmission line. The total discharge circuit inductance was $23 \mathrm{nH}$ when a $1 \mathrm{~mm}$ diameter, $1 \mathrm{~cm}$ long capillary was used. The capacitors and the transmission line were enclosed in an acrylic box filled with transformer oil to avoid external flashover. While this discharge has a slightly larger inductance than the one corresponding to the foil capacitor, it can be operated at voltages up to $40 \mathrm{kV}$, corresponding to peak currents of up to $60 \mathrm{kA}$ through a 1 $\mathrm{mm}$ diameter, $1 \mathrm{~cm}$ long capillary channel. The current pulses have a $10-90 \%$ risetime of $50 \mathrm{~ns}$ and a FWHM pulsewidth of $108 \mathrm{~ns}$ in the first half cycle.

In agreement with previous reports [13] it was found that polyethylene capillaries, unlike polyacetal, do not fracture at these discharge energies, and consequently they can be used for a large number of shots. Their diameters, however, are enlarged due to shock-wave compression and by ablation. For example, $1 \mathrm{~cm}$ long polyethylene capillaries having an initial diameter of $1 \mathrm{~mm}$ were found to expand to $1.020 \mathrm{~mm}$ and $1.125 \mathrm{~mm}$ when excited by a single pulse of 20 and $80 \mathrm{~J}$, respectively. Successive shots were observed to cause smaller increase of the capillary diameters. If caused solely by ablation, the measured en- 

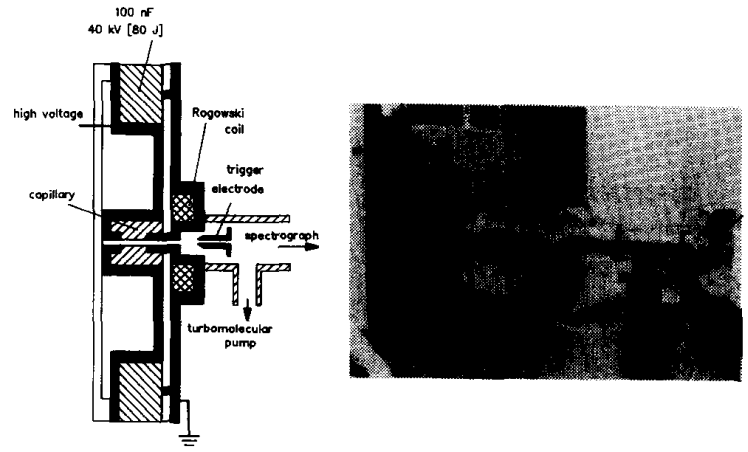

Fig. 2. Schematic diagram and photograph of the ceramic capacitor capillary discharge.

largement of the capillary volume for the $80 \mathrm{~J}$ discharge pulse would correspond to a mass loss of $1.9 \mathrm{mg}$. However, the difference in the weight of the capillary before and after the discharge shot was measured to be $1.1 \mathrm{mg}$. It is concluded that both shock wave compression and ablation contribute to the increase in capillary diameters, the first being increasingly important at high discharge energies. During the experiments described herein the polyethylene capillaries were replaced after several shots. Polyacetal capillaries often developed radial fractures after the first several discharge shots when operated at $15 \mathrm{kV}$, but could be utilized for a large number of shots when operated below $10 \mathrm{kV}$. The presence of these fractures significantly reduces the hold off voltage of the polyacetal capillaries, which, after being damaged, is usually only a few kilovolts. Both discharge structures were designed to allow for easy replacement of the capillaries by removing the anode electrode.

\section{Diagnostics System}

The axial emission from the capillary discharges was studied using a $1 \mathrm{~m}$ focal length grazing incidence vacuum spectrograph provided with a gatable multichannelplate intensified array detector that can be displaced tangentially to the Rowland circle. Two gold coated gratings with 300 and 1200 lines per millimeter were used at $86^{\circ}$ grazing incidence. The discharge radiation was focused into the slit of the spectrograph by a cylindrical aluminum mirror placed also at $86^{\circ}$ grazing incidence. A helium neon laser beam was directed through the capillary to align its axis with the spectrograph slit. Each spectrum was obtained in a single discharge shot, and the shot to shot reproducibility was good. Time resolved spectra were obtained by gating the multichannel-plate intensifier by applying a high voltage pulse. The excitation pulses to the multichannel-plate were generated by discharging coaxial cable transmission lines through a fast MOSFET, in a way similar to that described in [19]. The length of the gate pulse was controlled by selecting the length of the transmission lines, and the maximum temporal resolution was approximately $6 \mathrm{~ns}$.

The temporal evolution of the intensity of selected lines of interest was also measured utilizing the same instrument in the monochromator configuration, this time employing as detector a plastic scintillator-photomultiplier arrangement with a combined risetime of less than $4 \mathrm{~ns}$. The scintillator utilized was a Bicron $\mathrm{BC} 404$, which has a risetime of $0.7 \mathrm{~ns}$ and a FWHM pulsewidth of $2.2 \mathrm{~ns}$. The light produced by the plastic scintillator was coupled to the photomultiplier utilizing the optical fiber bundle. The photomultiplier signal was acquired with a dual channel 1 gigasample per second waveform digitizer. The second channel of the digitizer was used to simultaneously record the discharge current pulse.

\section{XUV Emission from Polyethylene Capillaries}

The XUV emission from polyethylene capillary discharges was studied as a function of the discharge voltage and time. The results discussed in this section were all obtained with the ceramic capacitor discharge. Fig. 3 shows the variations of the time-integrated emission from a $1 \mathrm{~mm}$ diameter $1 \mathrm{~cm}$ long polyethylene capillary discharge as a function of the discharge voltage in the spectral region extending from 14 to $24 \mathrm{~nm}$. At $10 \mathrm{kV}$ only line emission from $\mathrm{Cv}$ at $18.6 \mathrm{~nm}$ is clearly distinguished from the background of continuum radiation. For a $20 \mathrm{kV}$ discharge, corresponding to $20 \mathrm{~J}$ excitation, the CVI 3-2 line becomes dominant. Shorter wavelength spectra show that at this voltage the intensities of the Cvi $3.37 \mathrm{~nm}$ and the $\mathrm{Cv} 4.03 \mathrm{~nm}$ resonant lines are approximately equal. As shown in Fig. 3, the intensity of the Cvi $18.2 \mathrm{~nm}$ transition continues to increase as a function of the excitation voltage up to the maximum voltage explored, $35 \mathrm{kV}$, to become more than five times as intense as the neighboring $\mathrm{CV}$ and OVI lines. The comparison of the line intensity ratio between the CVI and the $\mathrm{Cv}$ lines in the time-integrated spectra of the $\mathrm{kV}$ discharge with collisional-radiative calculations (described below) indicate that the temperature reaches at least $66 \mathrm{eV}$.

The temporal evolution of the axial emission from a $25 \mathrm{kV} 40 \mathrm{kA}$ discharge is illustrated by the sequence of spectra shown in Fig. 4. The spectra have a temporal resolution of approximately $6 \mathrm{~ns}$. The circles in the current pulse indicate the relative time at which each of the spectra was taken. The evolution of the intensity of the CvI Balmer $\alpha$ and $\mathrm{Cv} 18.6 \mathrm{~nm}$ lines was also recorded utilizing the same instrument in the monochromator mode. These measurements show that the intensity of the CvI Balmer $\alpha$ line peaks in the range of 35 to $50 \mathrm{~ns}$ after the maximum of the current pulse and rapidly decreases as the current drops. The $\mathrm{Cv}$ line peaks later in time, as the temperature drops and $\mathrm{Cv}$ ions recombine. No Cvi emission was observed at the end of the first half cycle of the current pulse, the time at which a cooler plasma could generate a population inversion. A similar temporal evolution of the line intensities was also observed at higher discharge voltages, up to a maximum of $40 \mathrm{kV}$.

The ratio of the intensities of the 18.2 and $18.6 \mathrm{~nm}$ lines, which correspond to subsequent ionization stages 

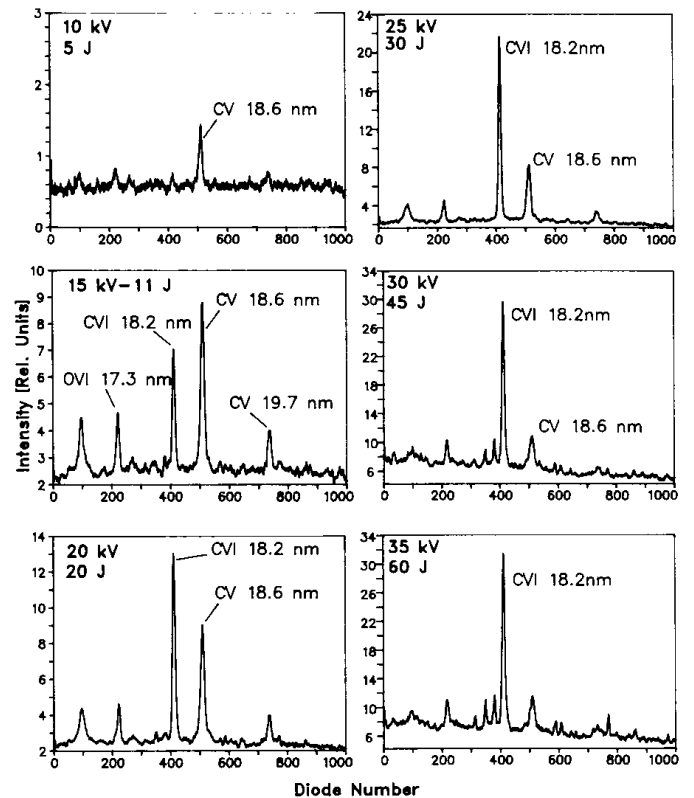

Fig. 3. Time-integrated axial emission from a $1 \mathrm{~mm}$ diameter $1 \mathrm{~cm}$ long polyethylene capillary discharge in the 14-24 $\mathrm{nm}$ spectral region for different discharge voltages.
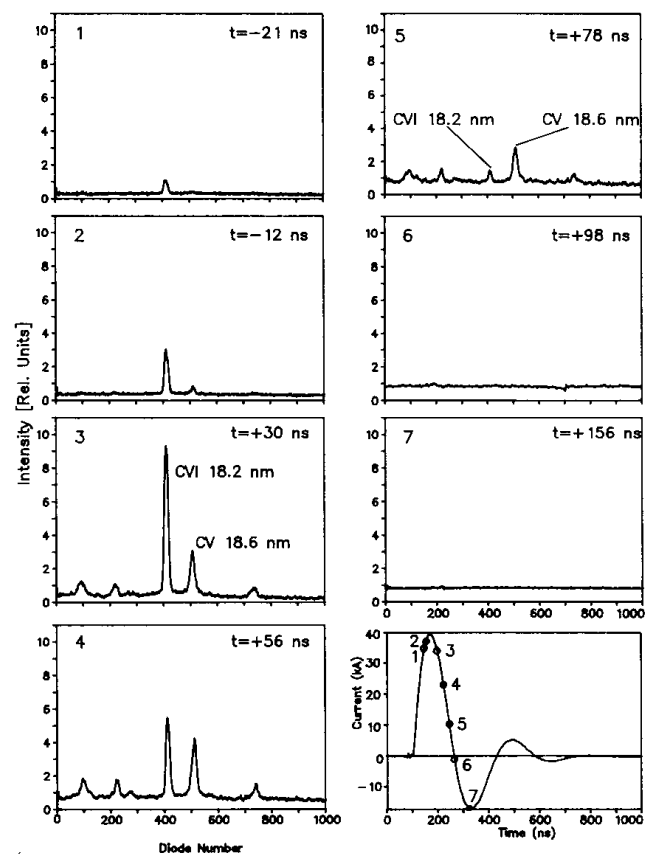

Fig. 4. Time resolved $14-24 \mathrm{~nm}$ spectra of $1 \mathrm{~mm}$ diameter $1 \mathrm{~cm}$ long polyethylene capillary excited by a $25 \mathrm{kV}$ discharge.

of carbon, was utilized to determine the electron temperature. The small wavelength separation between the two lines makes a spectral sensitivity calibration of the detection system unnecessary. To investigate the electron temperature evolution in a $25 \mathrm{kV}$ discharge, the line intensity ratios from the time-resolved spectra of Fig. 4 were com- pared to those predicted in a non-LTE (local thermal equilibrium) collisional-radiative calculation by the code RATION [20]. Contributions from the $4 d{ }^{3} D-2 p{ }^{3} P^{\circ}$ and $4 p$ ${ }^{1} P^{\circ}-2 s^{\prime} S \mathrm{Cv}$ transitions were added to calculate the intensity of the $18.6 \mathrm{~nm}$ line. Allowance was made for opacity effects in determining the population densities, as well as for absorption of the radiation in the direction of observation. The ratio of line intensities is quite insensitive to variations of electron density in the range of interest $\left(1-2.5 \cdot 10^{19} \mathrm{~cm}^{-3}\right)$. An electron density of $1.5 \cdot 10^{19}$ $\mathrm{cm}^{-3}$, which is compatible with the line broadening measurements discussed below, was assumed in computing the line intensity ratio. The line intensity ratios measured in the vicinity of the peak of the current pulse (spectra 2 and 3 in Fig. 4) correspond to electron temperatures in the range of $60-65 \mathrm{eV}$. Subsequently, during the decay of the current pulse, the temperature decreases. However, during this time the plasma is recombining, and the electron temperature can be lower than that inferred from the line intensity ratio by assuming non-LTE conditions. Therefore, the values displayed in Fig. 6 for time delays $\geq 20$ ns respect to the maximum current represent only an upper limit to the electron temperature. The plasma can be expected to continue cooling until the end of the current pulse, however at this time no Cvi emission is observed. This indicates that the decay rate of the current is slow compared to the loss rate of highly charged ions caused by diffusion and recombination and that a more rapidly decaying current pulse is required if collisional recombination of CVII ions is to be obtained at the end of the current pulse.

Fig. 5 displays a time-integrated spectrum in the wavelength region between 10.6 and $14.4 \mathrm{~nm}$ for a $1 \mathrm{~mm}$ diameter polyethylene capillary excited by a $30 \mathrm{kV} 45 \mathrm{~J}$ discharge pulse. Lines of the CVI Balmer series originated from levels with principal quantum numbers ranging from $n=4$ to $n=8$ are observed. The increasing broadening of the spectral linewidths with $n$ caused by the Stark effect can be observed, and provides an estimate of the electron density. The linewidths were determined by the best fit of calculated line profiles to the spectral lines. These calculated line profiles were obtained by the convolution of Lorentzian functions with an experimentally obtained instrumental line shape and with Gaussian profiles to account for Doppler broadening. The FWHM of the Balmer $\beta$ and $\delta$ lines is rather insensitive to electron collision broadening and ion dynamic field. The electron density was determined utilizing the expression for the linewidths in the quasi-static theory as given by Griem [21], assuming the mean value of the charge of the perturbing ions to be 3.85 . This mean value for the charge was estimated according to [22] by assuming that the hydrogen is completely ionized, that the dominant carbon specie is $\mathrm{CVI}$, and considering that in polyethylene there are two hydrogen atoms per each carbon atom. The electron density that results from the Balmer $\beta$ and $\delta$ lines is $1.5 \cdot 10^{19} \mathrm{~cm}^{-3}$ and $1.3 \cdot 10^{19} \mathrm{~cm}^{-3}$, respectively. This electron density is close to the value required for the amplification of the 


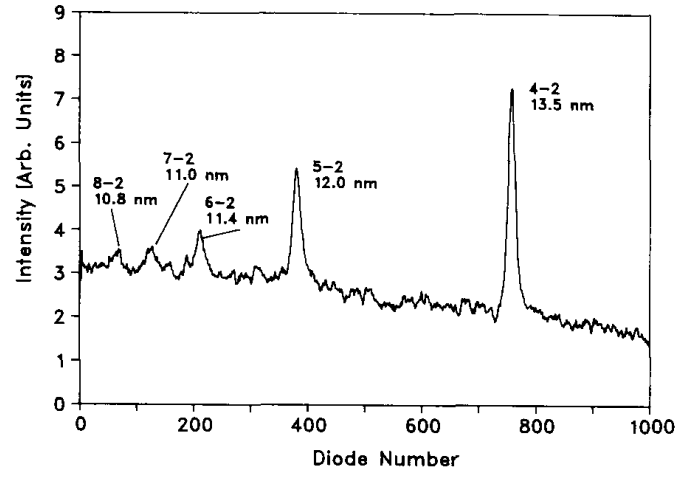

Fig. 5. Time-integrated spectrum of the Balmer series corresponding to $30 \mathrm{kV}, 45 \mathrm{~J}$ excitation of a $1 \mathrm{~mm}$ diameter $1 \mathrm{~cm}$ long polyethylene capillary.

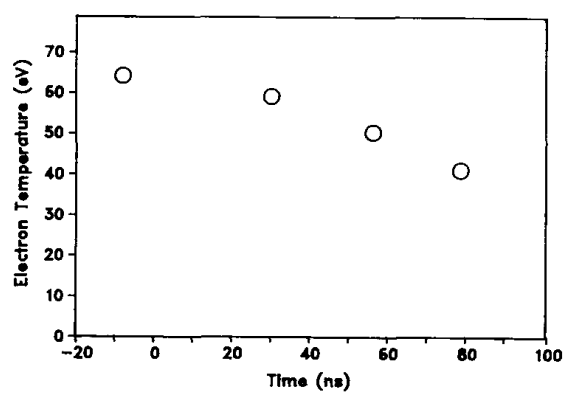

Fig. 6. Electron temperature evolution in a $1 \mathrm{~mm}$ diameter $1 \mathrm{~cm}$ long polyethylene capillary discharge excited by a $25 \mathrm{kV}$ discharge pulse as determined from the intensity ratio of the CVI $18.2 \mathrm{~nm}$ and $\mathrm{Cv} 18.6 \mathrm{~nm}$ lines. The origin of the time scale corresponds to the peak of the current pulse. The values for time delays $\geq 20 \mathrm{~ns}$ (decay of the current pulse) represent an upper limit to the electron temperature.

Balmer $\alpha$ line of hydrogenic carbon by collisional recombination. Electron density values which are up to $20 \%$ larger result from allowing a correction that estimates the effect of electron Debye screening and ion-ion interaction [23]. Spectra obtained for other discharge shots at the same discharge conditions have however yielded a larger spread of values, including densities up to $2 \cdot 10^{19} \mathrm{~cm}^{-3}$. Time resolved spectra corresponding to times in the proximity of the peak of the Cvi line intensity did not show significantly different linewidths. This observation is probably related to the fact that the majority of the $\mathrm{CVI}$ line emission occurs in a relatively narrow time interval in which the plasma density does not change significantly.

To investigate the possibility of amplification of the $18.2 \mathrm{~nm}$ CVI 3-2 transition we compared the ratio of the intensity of the 4-2 transition in the same ion for different lengths of capillary plasma columns. To be able to make a valid comparison of the relative variation of line intensities as a function of the plasma length it is necessary to maintain the plasma conditions constant and therefore to preserve the characteristics of the discharge current pulse when the length of the plasma column is varied. This was achieved by selecting the diameter of the discharge electrodes to compensate for the increase of inductance as-
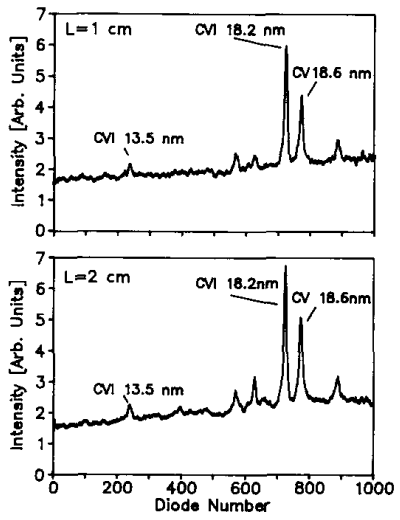

(a)

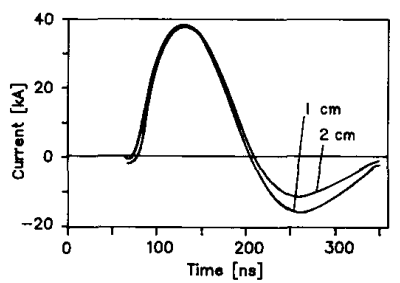

(b)

Fig. 7. (a) Time-integrated spectra corresponding to 1 and $2 \mathrm{~cm}$ long discharges through $1 \mathrm{~mm}$ diameter polyethylene capillaries excited by a 38 kA current pulse. (b) Discharge current pulses corresponding to the spectra shown in (a).

sociated with a longer plasma channel and by simultaneously increasing the discharge voltage to compensate for the larger resistance of the longer plasma column. Fig. 7 (a) shows the time integrated spectra corresponding to 1 and $2 \mathrm{~cm}$ long discharges through $1 \mathrm{~mm}$ diameter polyethylene capillary channels excited by the nearly identical first half cycle current pulses shown in Fig. 7(b). The ratio of the line intensities is very similar in both discharges, indicating that no amplification is observed.

\section{XUV Emission from Polyacetal Capillaries}

Experiments were also conducted in polyacetal capillaries 0.5 and $1 \mathrm{~mm}$ diameter with lengths varying from 1 to $2 \mathrm{~cm}$. The study of the soft X-ray emission from polyacetal capillary discharges is of interest in relation to the recently reported observation of short bursts of $18.2 \mathrm{~nm}$ radiation in capillaries of this material at discharge voltages as low as $7 \mathrm{kV}$ [18]. In that experiment, the soft X-ray emission from the polyacetal capillary plasmas was monitored with a monochromator supplied with a scintillator-photomultiplier detector. The short bursts in the photomultiplier signal, which were observed to occur at the time near the peak of the second half cycle of the current pulse and to increase in intensity with the capillary discharge length, were attributed to amplified spontaneous emission in the Cvi Balmer $\alpha$ line. Most of the results in polyacetal reported herein were obtained utilizing the foil capacitor discharge described in Section II that 
has a comparable capacitance and inductance to that of the discharge used in [18].

Fig. 8 illustrates the variation of the XUV emission of a $0.5 \mathrm{~mm}$ diameter, $1 \mathrm{~cm}$ long polyacetal capillary in the spectral region between 16.5 and $21 \mathrm{~nm}$ as a function of the discharge voltage. At $7 \mathrm{kV}$ only oxygen lines were observed. The Cvi Balmer $\alpha$ line was detected when the discharge voltage was increased above $10 \mathrm{kV}$. These time integrated spectra are in good agreement with those recently reported by Morgan et al. [24]. The CVI emission remained weak compared to the emission from the $17.3 \mathrm{~nm}$ Ovi line even when the discharge voltage was increased to $25 \mathrm{kV}$, the maximum value at which we could operate the foil capacitor discharge. Polyacetal spectra obtained operating the ceramic capacitor discharge at $30 \mathrm{kV}$ also showed weak Cvi emission.

Data obtained for $1 \mathrm{~mm}$ diameter polyacetal capillaries showed a similar trend in the line intensities as a function of voltage, but shifted towards higher voltages. For example, the characteristics of the time integrated spectra obtained for the larger diameter discharge at $20 \mathrm{kV}$ are similar to those obtained for the $0.5 \mathrm{~mm}$ capillary at $15 \mathrm{kV}$. When the length of the capillaries was increased to $2 \mathrm{~cm}, 18.2 \mathrm{~nm} \mathrm{CvI} \mathrm{emission} \mathrm{was} \mathrm{observed} \mathrm{to} \mathrm{be} \mathrm{weaker}$ than in $1 \mathrm{~cm}$ long capillary excited by the same discharge voltage. The decreased CvI emission in the $2 \mathrm{~cm}$ discharges is associated with a smaller discharge current caused by the increase in the inductance of the discharge circuit for the longer capillary channel.

Time resolved spectra were obtained by gating the multichannel plate intensifier to integrate the emitted radiation during temporal windows of either 6 or $50 \mathrm{~ns}$. The larger window was utilized in combination with adjustable time delays to monitor the emission during the entire first cycle of the current pulse. This relatively large time window was selected to be able to observe the possible existence of an intense burst of line radiation that might appear with a jitter of a few tens of nanoseconds relative to the current pulse. The $6 \mathrm{~ns}$ gating time window was utilized to be able to detect the possible existence of temporally narrow bursts of line radiation that might have a small integrated intensity as compared with the continuum background radiation.

Figs. 9 and 10 display sequences of temporally resolved spectra corresponding to polyacetal capillaries 0.5 and $1 \mathrm{~mm}$ diameter, respectively, both $1 \mathrm{~cm}$ in length, excited by a $16 \mathrm{kV}$ discharge. The position and width of the time window of each spectrum relative to the current pulse is indicated in the lower part of the figures. Line emission is observed to be strong only during the first half cycle of the current pulse, and at all times it is dominated by the Ovi $17.3 \mathrm{~nm}$ radiation. The Cvi emission, which is stronger in the $0.5 \mathrm{~mm}$ diameter discharges, is observed in both cases to peak shortly after the maximum of the current pulse and to completely vanish by the end of the first half cycle of the current pulse. This shows, as already discussed for the polyethylene discharges, that the current decay rate is slow compared with the loss rate of highly
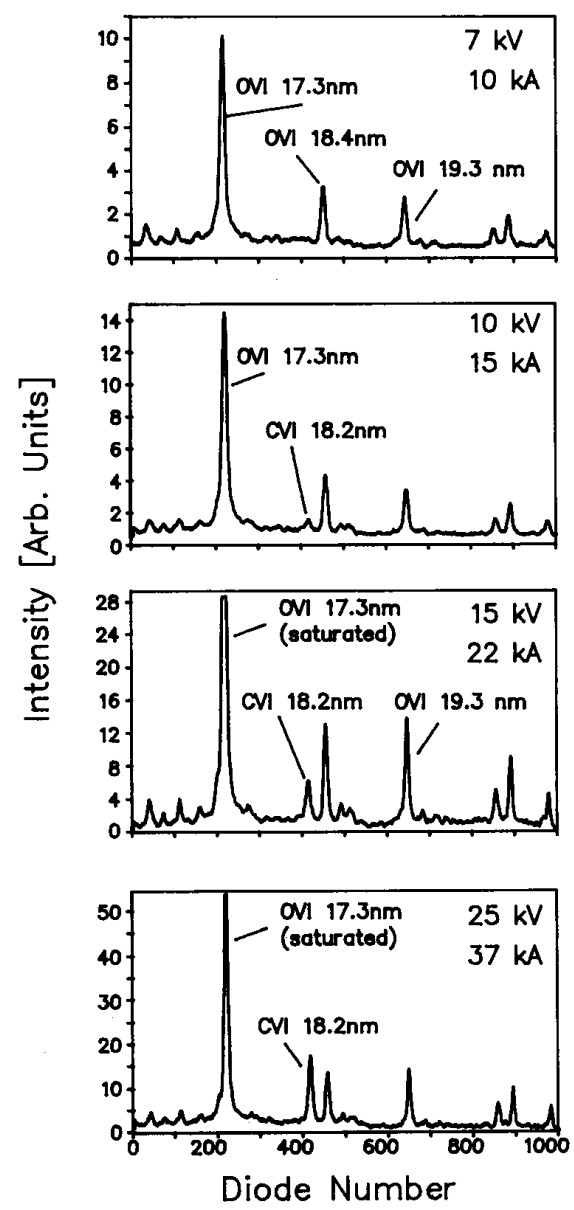

Fig. 8. Time integrated $16.5-21 \mathrm{~nm}$ spectra from a $0.5 \mathrm{~mm}$ diameter $1 \mathrm{~cm}$ long polyacetal capillary as a function of the discharge voltage.
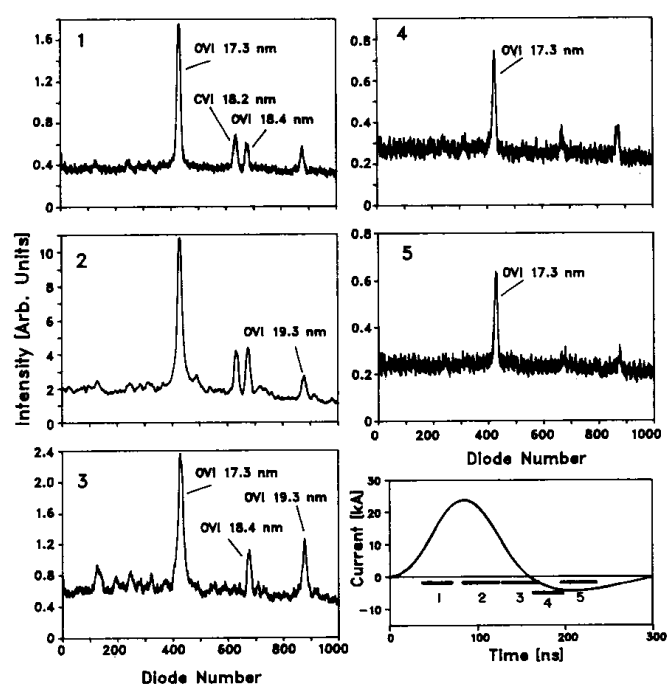

Fig. 9. Sequence of time resolved spectra of the emission from a $0.5 \mathrm{~mm}$ diameter, $1 \mathrm{~cm}$ long polyacetal capillary excited by a $16 \mathrm{kV}$ voltage pulse. The timing of each spectrum relative to the current pulse is indicated. 

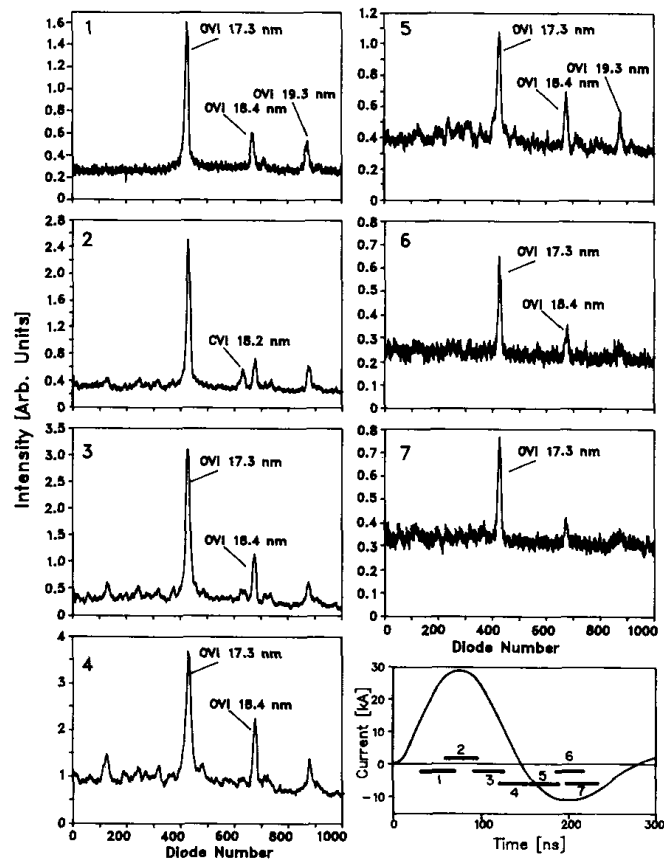

Fig. 10. Sequence of time resolved spectra of the axial emission from a $\mathrm{mm}$ diameter $1 \mathrm{~cm}$ long polyacetal capillary excited by a $16 \mathrm{kV}$ voltage pulse. The timing of each spectrum relative to the current pulse is indicated.

charged carbon ions due to diffusion and recombination. During the second half cycle of the current pulse the increased mass accumulated in the plasma from the continuous ablation of the walls and the decreased magnitude of the excitation results in a cooler plasma with insignificant radiation originating from highly ionized species.

The time-resolved spectra obtained activating the detector for $\mathbf{6} \mathrm{ns}$ time windows are in good agreement with those obtained with the wider temporal windows for similar time delays. A large number of spectra obtained with the narrower temporal window at times corresponding to the second half cycle of the current pulse were unable to identify line emission bursts at $\mathbf{1 8 . 2} \mathrm{nm}$ and only showed weak radiation from Ovi lines.

The temporal evolution of the soft X-ray emission at several selected wavelengths was also measured operating the instrument in the monochromator mode and utilizing as detector the scintillator-photomultiplier combination previously described in Section III. Fig. 11 shows the temporal evolution of the $18.2 \mathrm{~nm}$ emission relative to the current pulse for a $0.5 \mathrm{~mm}$ diameter $1 \mathrm{~cm}$ long polyacetal capillary excited by a $16 \mathrm{kV}$ pulse. The intensity of the radiation at this wavelength was observed to peak shortly after the maximum of the current pulse in agreement with the time resolved spectra of Fig. 9. Similar measurements conducted in $2 \mathrm{~cm}$ long polyacetal capillaries of the same diameter and in capillaries $1 \mathrm{~mm}$ in diameter having 1 and $2 \mathrm{~cm}$ in length were also unable to identify burst of radiation at $18.2 \mathrm{~nm}$. While for very high gain settings of the photomultiplier tube narrow spikes in the signal were ob-

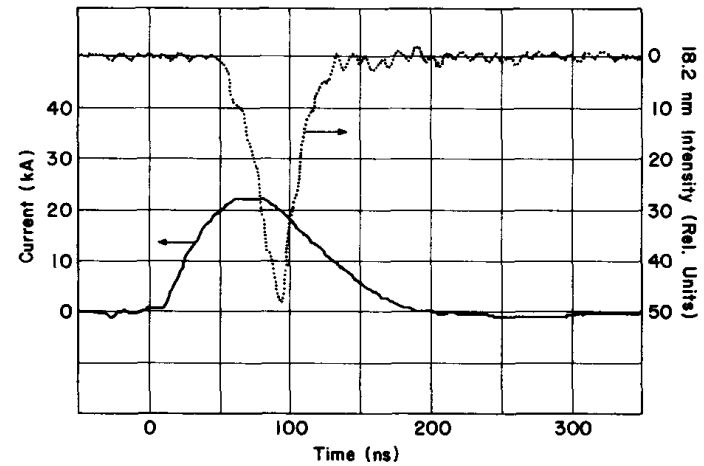

Fig. 11. Temporal evolution of the $18.2 \mathrm{~nm}$ emission from a $0.5 \mathrm{~mm}$ diameter $1 \mathrm{~cm}$ long polyacetal capillary excited by a $16 \mathrm{kV}$ discharge. The current pulse is also shown.

served, these spikes occurred at several wavelength settings of the monochromator and identify well with electron pulses caused by the multiplication that follows single photocathode emission events.

In summary, the soft X-ray emission in polyacetal capillaries in the discharge regime investigated is dominated by Ovi lines, and the Cvi emission is weak compared to that generated in polyethylene capillaries. Within the range of the discharge parameters utilized in the experiments reported herein, the electron temperature of these discharges seems too low to allow for the generation of the Cvil concentration required to obtain measurable amplification from collisional recombination in CVI. The increased radiation loss caused by the presence of oxygen in the polyacetal capillaries is likely to be responsible for the formation of a cooler plasma with reduced Cvi emission.

\section{High Energy Beam Electrons in Capillary DisCHARGES}

High voltage discharges in vacuum or at low pressure are known to generate intense beams of high energy electrons [25]-[27]. Repeated shots from a conically tapered capillary discharge have been previously observed to produce a small diameter hole in the anode [13]. These perforations suggest the presence of fast particle beams.

To detect the possible presence of a stream of high energy electrons that could contribute to the generation of the highly ionized species observed in the XUV spectra, a Faraday cup was installed behind the perforated anode electrode of the capillary discharge. The setup utilized in this experiment is schematically illustrated in Fig. 12(a). The measurements were performed in a polyethylene capillary $1 \mathrm{~mm}$ diameter and $1 \mathrm{~cm}$ in length. The Faraday cup was placed behind a grounded mesh of $63 \%$ transmissivity which could be negatively biased to reject low energy electrons. The Faraday cup was made out of graphite, having a concave conical shape to minimize the emission of secondary electrons. The secondary electron current was measured to be insignificant. All data were obtained from untriggered discharges to avoid the detec- 


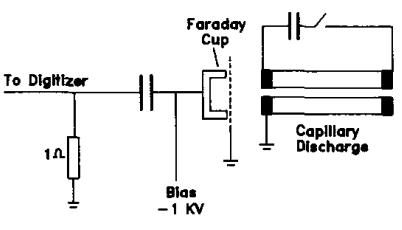

(a)

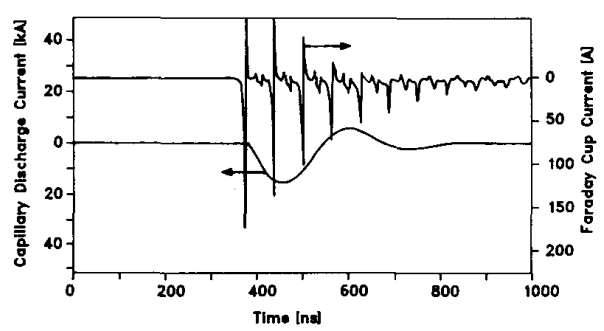

(b)

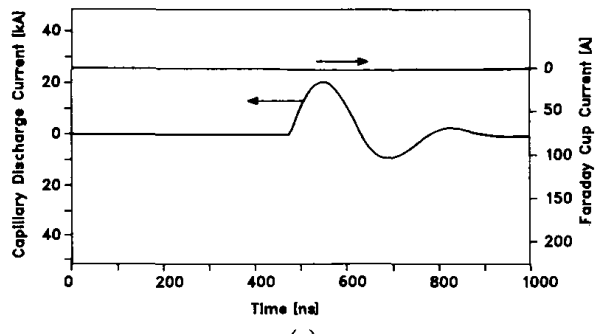

(c)

Fig. 12. (a) Faraday cup setup utilized to detect energetic beam electrons in a capillary discharge. (b) Current pulse collected by the Faraday cup corresponding to a $23 \mathrm{kA} 1 \mathrm{~mm}$ polyethylene capillary discharge. The discharge capacitors were negatively charged such that the discharge electrode facing the cup was the anode. The current pulse is also shown. The Faraday cup was unbiased, and the distance from the cup to the anode was $1.8 \mathrm{~cm}$. (c) Current pulse collected by the Faraday cup when the discharge capacitors were positively charged. In this case the discharge electrode facing the cup is the cathode.

tion by the cup of any fast particle that could be associated with the trigger pulse. The current collected by the Faraday cup was measured across a $1 \Omega$ resistor using a dualchannel 1 gigasample per second waveform digitizer placed inside a Faraday cage. The second channel was utilized to simultaneously record the current pulse flowing through the capillary.

Short burst of high energy electrons were detected in coincidence with the onset of the discharge current pulse. However, unneutralized high-current density electron beams do not easily propagate in an equipotential region [28] such as the space between the grounded anode of the discharge and the Faraday cup, and consequently both the amplitude and the pulse shape of the electron pulses detected by the Faraday cup were found to vary from one discharge shot to the next. Increased reproducibility in the measurements was obtained by injecting a small amount of helium in the discharge to allow for charge neutralization [29]. Fig. 12(b) shows the current detected by the unbiased Faraday cup in a capillary discharge generated by charging the capacitors negatively, situation in which the grounded discharge electrode facing the Faraday cup is the anode of the discharge during the first half cycle of the current pulse. The first peak in the Faraday cup signal of Fig. 12(b), which occurs shortly after the initiation of the $16 \mathrm{kA}$ discharge current pulse, is a burst of electrons having approximately $5 \mathrm{~ns}$ FWHM pulsewidth and a peak current of $180 \mathrm{~A}$. The subsequent peaks in Fig. 12(b) are reflections of this signal at the end of the $50 \Omega$ cable utilized to transmit the Faraday cup signal to the waveform digitizer. When the Faraday cup was negatively biased to $-1 \mathrm{kV}$ to reject slow electrons, the peak of the collected electron current remained above $150 \mathrm{~A}$.

As further verification that the signal collected by the Faraday cup is due to beam electrons, the polarity of the high voltage electrode in the capillary discharge was reversed. In this situation, electrons are accelerated in the direction opposite to the Faraday cup, and the collected signal should be drastically reduced. In effect, Fig. 12(c) shows that the signal detected by the unbiased Faraday cup was negligible when the capillary discharge capacitors were positively charged.

Fig. 13(a) shows the electron current collected as a function of the distance from the end of the capillary discharge to the Faraday cup. The plot shows that the current collected at distances smaller than $2.5 \mathrm{~cm}$ is almost constant and consequently the value for $1.8 \mathrm{~cm}$ can be considered a good estimate of the electron beam current existing at the anode-end of the capillary channel. Fig. 12(b) shows that the electron current collected by the Faraday cup increases as a function of the capillary discharge current. The maximum discharge current shown in the figure, $17.5 \mathrm{kA}$, was limited by the breakdown voltage that could be obtained at a pressure of 1 torr of helium, injected to allow for electron beam neutralization. Measurements at higher discharge currents were done reducing the helium pressure at 20 mtorr. At a discharge current of $40 \mathrm{kA}$, Faraday cup current pulses up to $1200 \mathrm{~A}$ and $5 \mathrm{~ns}$ pulsewidth were measured near the onset of the discharge.

In summary, a stream of fast electrons was detected. However, it occurs at the onset of the discharge, shortly after surface flashover, when the majority of the capillary volume is not filled by plasma. At that instant the $E / N$ ratio between the applied electric field and the density of atoms in the capillary channel is large, and the long mean free path between electron collisions results in the generation of a large number of runaway electrons. The beam has a very short duration ( $5 \mathrm{~ns}$ ) as a consequence of the rapid increase in the density of atoms in the discharge due to the ablation of material from the capillary walls. The energy of these short electron pulses is not larger than few tens of millijoules and is small compared with the energy dissipated in the discharge by ohmic heating. Since energetic beam electrons are detected only 80-100 ns before the peak of the CVI emission, these electrons could only contribute to the excitation of the observed Cvi emission through the generation of CVII ions that could later recombine. However, the time history of the carbon ion soft $\mathrm{X}$-ray emission in polyethylene discharges tends to disprove this possibility, since it shows that the peak of the Cvi line radiation, which occurs shortly after the maxi- 


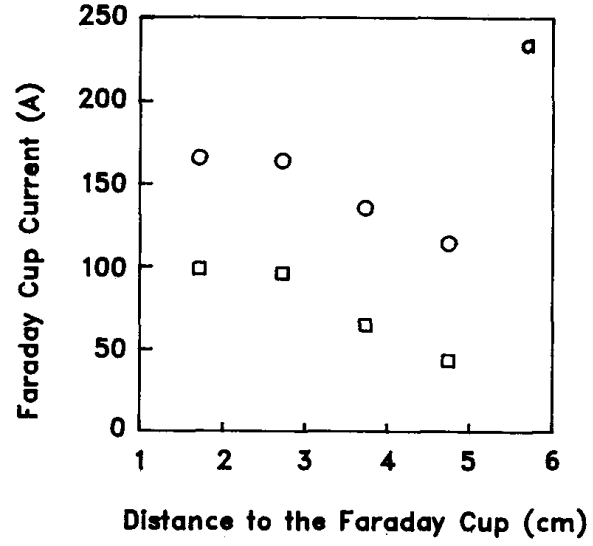

(a)

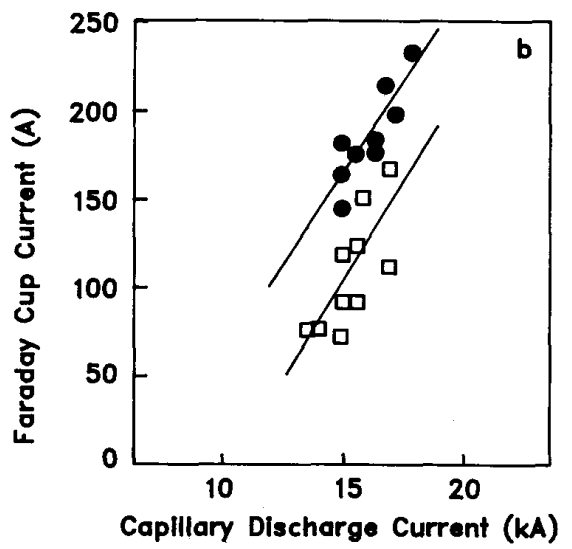

(b)

Fig. 13. (a) Peak current collected by the Faraday cup as a function of the distance between the cup and the end of the capillary in a $17 \mathrm{kA} 1 \mathrm{~mm}$ diameter polyethylene capillary. $\bigcirc-$ unbiased cup, $\square-1 \mathrm{kV}$ bias. (b) Peak current collected by the Faraday cup as a function of the peak value of capillary discharge current pulse. - unbiased cup, $\square-1 \mathrm{kV}$ bias.

mum of the current pulse, is preceded by $\mathrm{Cv}$ line radiation.

\section{Summary and Continuing Work}

The soft $\mathrm{X}$-ray emission from polyethylene and polyacetal capillary discharges excited by current pulses having approximately $100 \mathrm{~ns}$ FWHM, a $50 \mathrm{~ns}$ risetime and peak currents up to $60 \mathrm{kA}$ has been studied. Intense CVI line emission was observed to dominate the spectra of $1 \mathrm{~mm}$ diameter polyethylene discharges for excitation currents above $35 \mathrm{kA}$. In contrast, emission of polyacetal capillaries was found to be dominated by the presence of oxygen, and to show only weak $18.2 \mathrm{~nm} \mathrm{CVI}$ radiation. The plasma density in a $1 \mathrm{~mm}$ diameter polyethylene discharge excited by a $50 \mathrm{kA}$ current pulse was found, from Stark broadening of $\mathrm{CVI}$ Balmer lines, to be in the range of $1.3 \cdot 10^{19} \mathrm{~cm}^{-3}$ to $2 \cdot 10^{19} \mathrm{~cm}^{-3}$. Line intensity ratios of $\mathrm{CVI}$ and $\mathrm{Cv}$ lines indicate that the peak electron temperature, and consequently the degree of ionization, in- crease as a function of discharge voltage in the range investigated, reaching $66 \mathrm{eV}$ for a $35 \mathrm{kV} 57 \mathrm{kA}$ discharge.

The possibility that the highly ionized particles might be generated by beam electrons that constitute the most energetic part of a two-temperature electron energy distribution was investigated. A short burst of energetic $(E>1 \mathrm{keV})$ electrons was detected. However, it occurs in coincidence with the onset of the discharge current pulse, a time at which the $E / N$ ratio is high and the majority of the capillary volume is not filled by plasma. No current of beam electrons was detected later in the current pulse when the CVI radiation is observed.

The Cvi emission in both the polyethylene and polyacetal discharges was observed to peak shortly after the maximum of the current pulse and to vanish before the end of the first half cycle of the current pulse, indicating that in these discharges the decay rate of the current pulse is slow compared with the loss rate of the highly charged ions caused by diffusion and recombination. Most of the highly charged $\mathrm{CvI}$ ions generated in the plasma core are therefore lost at a time when the recombination rate is still limited by the ohmic heating caused by the current flow. A more rapid discharge is required if recombination of CVII ions is to occur at the end of the current pulse. Also cooling to a temperature below $20 \mathrm{eV}$ is necessary if significant gain is to be obtained. It is foreseeable that population inversions and gain could nevertheless occur in the type of discharges discussed herein during the decay of the current pulse and before the plasma core has cooled significantly, if highly ionized ions from the plasma core diffuse and recombine into the cooler boundary region. It has been previously suggested [15], [16] that the diffusion of totally ionized atoms from the core plasma into the cooler boundary plasma might lead to an annular region of high recombination, similar to that observed in laser created plasmas [2], in which soft X-ray amplification might occur under optimized plasma conditions. However, the comparison of the soft X-ray spectra of 1 and $2 \mathrm{~cm}$ capillaries excited by identical current pulses has shown no amplification at $\mathbf{1 8 . 2} \mathrm{nm}$ for the discharge conditions investigated.

To achieve initially hotter and more rapidly cooled plasmas we have developed a fast high power density discharge having a 10-90\% current risetime of $11 \mathrm{~ns}$, a similar fall time, and a FWHM pulsewidth of $25 \mathrm{~ns}$. When operated at $600 \mathrm{kV}$ this discharge has produced a current peak of $140 \mathrm{kA}$ through a $1 \mathrm{~mm}$ diameter $1 \mathrm{~cm}$ long capillary. The fast rise time of the capillary discharge current should allow for the generation of hotter plasmas [30]. A rapid current risetime can cause the magnetic confinement to build up faster than the plasma pressure, producing a compressed plasma column with increased temperature and a higher degree of ionization. Also, the faster current fall time should allow for a lower plasma temperature at a time when a high density of CVII ions is available for recombination as required to achieve maximum gain with recombination pumping. The first soft X-ray spectra of polyethylene plasmas excited by this fast discharge show 
strong Cvi emission at the end of the first half cycle of the current pulse, in contrast with the results reported herein for the slower discharges. A study of the plasma column generated by this fast discharge is currently being carried out and will be reported in a future publication.

\section{ACKNOWLEDGMENT}

We want to thank O. Buccafusca, K. Richardson, and B. Szapiro for their collaboration in the design and construction of the capillary discharges, and Dr. R. W. Lee for making a copy of his code RATION available.

\section{REFERENCES}

[1] D. L. Matthews, P. L. Hagelstein, M. D. Rosen, M. J. Eckart, N M. Ceglio, A. U. Hazi, H. Medecki, B. MacGowan, J. E. Trebes, B. L. Whitten, E. M. Campbell, C. H. Hatcher, A. M. Hawtyluk, R. L. Kauffman, L. D. Pleasance, G. Rambach, J. H. Scofield, G Stone, and T. Weaver, "Demonstration of soft X-ray amplifier,' Phys. Rev. Lett., vol. 54, p. 110, 1985.

[2] S. Suckewer, C. H. Skinner, H. Milchberg, C. Keane, and D. Voorhees, "Amplification of stimulated emission in a confined plasma column," Phys. Rev. Lett., vol. 55, p. 1753, 1985.

[3] C. Chenais-Popovics, R. Corbett, C. J. Hooker, M. H. Key, G. P. Kiehm, C. L. S. Lewis, G. J. Pert, C. Regan, S. Sadaat, T. Tomie, and D. Willi. "Laser amplification at $18.2 \mathrm{~nm}$ in recombining plasma from a laser irradiated carbon fibre," Phys. Rev. Lett., vol. 59, p. $2161,1987$.

[4] T. N. Lee, E. A. McLean, and R. C. Elton. "Soft X-ray lasing in neon-like germanium and copper plasmas," Phys. Rev, Lett., vol. 59, p. $1185,1987$.

[5] D. L. Matthews, "The soft X-ray laser, present status and future prospects," Bull. Amer. Phys. Soc., vol. 33, p. 2041, 1988.

[6] S. Suckewer, C. H. Skinner, D. Kim, E. Valeo, D. Voorhees, and A. Wouters, "Divergence measurements of a soft X-ray laser beam," Phys. Rev. Lett., vol. 57, p. 1004, 1986.

[7] J. E. Trebes, S. B. Brown, E. M. Campbell, D. L. Matthews, D. G. Nilson, G. F. Stone, and D. A. Whelan, "Demonstration of X-ray holography with an X-ray laser," Science, vol. 238, p. 517, 1987.

[8] D. S. DiCicco, D. Kim, R. J. Rosen, C. H. Skinner, S. Suckewer, A. P. Gupta, and J. G. Hirschberg, "Contact microscopy with a soft $\mathrm{X}$-ray laser," OSA Proc. on Short Wavelength Coherent Radiation, Generation and Applications, F. Falcone and J. Kirz, Eds., vol. 2, p. 277,1988 .

[9] P. L. Hagelstein, "Development of the MIT table-top soft X-ray laser," Proc. SPIE, vol. 1551, July 1991.

[10] D. E. Kim, C. A. Skinner, D. Voorhees, and S. Suckewer. "Development of small scale soft X-ray lasers,"' Proc. SPIE, vol. 1551, July 1991

[11] J. J. Rocca, D. C. Bethe, and M. C. Marconi, "Proposal for soft X-ray and XUV lasers in capillary discharges," Opt. Lett., vol. 13, pp. $565,1988$.

[12] P. Bogen, H. Conrads, G. Gatti, and W. Kohlhaas. "Continuum radiation source of high intensity,'” J. Opt. Soc. Amer., vol. 58, p. 203 1968.

[13] R. A. McCorkle, "The high power sliding spark capillary discharge in vacuum: Variations and applications," Appl. Phys. A, vol. 26, p. 261,1981 .

[14] S. M. Zakharov, A. A. Kolomenskii, S. A. Pikuz, and A. I. Samokhin. "Soft X-ray emission from a high current capillary discharge," Sov. Tech. Phys. Lett., vol. 6, p. 486, 1980.

[15] M. C. Marconi and J. J. Rocca, "Time resolved extreme ultraviolet emission from a highly ionized lithium capillary discharge," Appl. Phys. Lett., vol. 54, p. 2180, 1989.

[16] J. J. Rocca, M. C. Marconi, D. Bethe, and M. Villagran Muniz. "Prospects of XUV recombination lasers in capillary discharges," Proc. Int. Conf. on Lasers '88, R. C. Sze and F. J. Duarte, Eds. McLean, VA: STS, 1989, pp. 278-285.

[17] R. C. Elton, "Progress and trends in X-ray laser research," Opt. Eng., vol. 21, p. 307, 1982

[18] C. Steden and H. J. Kunze, "Observation of gain at $18.2 \mathrm{~nm}$ in the carbon plasma of a capillary discharge," Phys. Lett. A, vol. 151, p. $534,1990$.

[19] M. C. Marconi, J. J. Rocca, and G. J. Krause. "Fast gating of a windowless dual-multichannel-plate intensified array detector," $J$. Phys. E: Sci. Instrum., vol. 22, p. 849, 1989.

[20] R. W. Lee, B. L. Whitten, and R. E. Strout, J. Quantum Spect. Radiat. Transfer, vol. 32 , p. 91,1984 . Use of a collisional-radiative code developed by the authors (J. Hung, J. Meyer, and J. J. Rocca, unpublished) yields similar results.

[21] H. R. Griem, Spectral Line Broadening by Plasmas. New York: Academic 1974.

[22] R. J. Tighe, and C. F. Hooper, Jr. "Low-frequency electron microfield distributions in a plasma containing multiply-charged ions: Extended calculations,"' Phys. Rev. A, vol. 15, p. 1773, 1977.

[23] G. Bekefi, C. Deutsch, and B. Yaakobi, "Spectroscopic diagnostics of laser plasmas," in Principles of Laser Plasmas, G. Bekefi, Ed. New York: Wiley, 1976.

[24] C. A. Morgan, E. Iglesias, H. R. Griem, H. J. Kunze, and R. C. Elton, "A capillary discharge experiment for the investigation of possible gain in the $\mathrm{H} \alpha$ line of," presented at IEEE Int. Conf. Plasma Science, June 3-5, 1991, Williamsburg, VA, paper 3P44

[25] P. Choi, H. H. Chuaqui, M. Favre, and E. S. Wyndham, “An observation of energetic electron beams in low pressure linear discharges," IEEE Trans. Plasma Sci., vol. PS-15, p. 438, 1987.

[26] J. Christiansen and C. Schultheiss, "Production of high current particle beams of low pressure spark discharges, ' Z. Physik, vol. A290, p. 36, 1979.

[27] H. F. Ranea-Sandoval, N. Reesor, B. T. Szapiro, C. Murray, and J. J. Rocca, "Study of intense electron beams produced by high voltage glow discharges," IEEE Trans. Plasma Sci., vol. PS-15, p. 361, 1987.

[28] J. R. Pierce, Theory and Design of Electron Beams. NJ: Van Nostram, 1954.

[29] J. R. Smith, R. F. Schneider, M. J. Rhee, H. Suhm, and W. Namkung, "Propagation of a mildly relativistic electron beam at subtorr pressures," J. Appl. Phys., vol. 60, p. 12, 1986.

[30] R. C. Elton, X-Ray Lasers. San Diego, CA: Academic, 1990

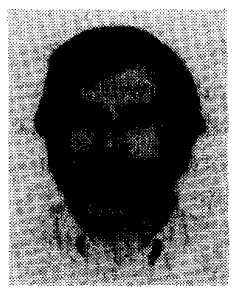

Jorge J. Rocca (S'80-M'83) received the diploma in physics from the University of Rosario, Argentina, in 1978 and the Ph.D. degree from Colorado State University, Fort Collins, in 1983. $\mathrm{He}$ is currently a Professor with the Department of Electrical Engineering, Colorado State University. His broad research interests include the development of new short-wavelength lasers and the physics of discharges and plasmas. His work has included the development of ion lasers excited by electron beams, the generation of intense electron beams from glow discharges, the demonstration of recombination lasing in negative glow plasmas and the study of dense capillary discharges which he has proposed as possible source of $\mathrm{X}$-ray and XUV recombination laser radiation.

Dr. Rocca received a National Science Foundation Presidential Young Investigator Award for 1985-1990 and he is also a recipient of the Halliburton Foundation Award. He is a member of the Optical Society of America and the American Physical Society.

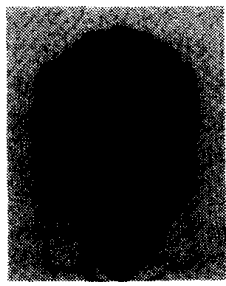

Mario C. Marconi was born in Buenos Aires, Argentina, on March 7, 1955. He received the degrees of Licenciado en Fisica and Doctor en Fisica from the Universidad de Buenos Aires in $\mathbf{1 9 8 0}$ and 1985 , respectively.

He joined CEILAP (CITEFA) in 1978, where he worked in new techniques to obtain short pulse emission in solid-state lasers. Since 1986 he has been with Colorado State University: Fort Collins, under a post-Doctoral Fellowship from the Universidad de Buenos Aires and the Consejo Nacional de Investigaciones Cientificas y Tecnicas (CONICET).

F. G. Tomasel, photograph and biography not available at the time of publication. 